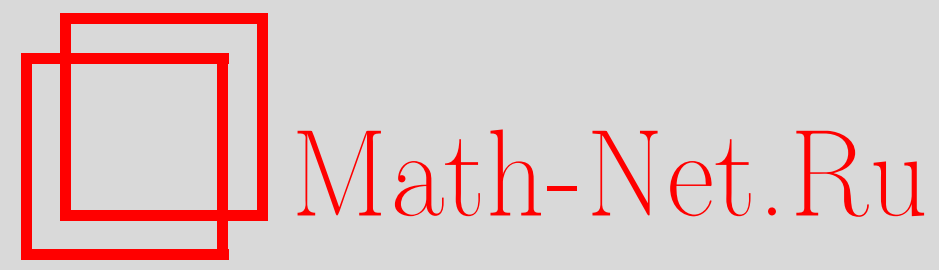

Ю.В.Медницкий, О существовании сильных решений линейных стохастических дифференциальных уравнений на $R^{\infty}$, Теория вероятн. и ее примен., 1997, том 42, выпуск 4, 826-831

DOI: https://doi.org/10.4213/tvp2615

Использование Общероссийского математического портала Math-Net.Ru подразумевает, что вы прочитали и согласны с пользовательским соглашением http://www . mathnet.ru/rus/agreement

Параметры загрузки:

IP : 54.147 .182 .235

26 апреля 2023 г., 15:21:45

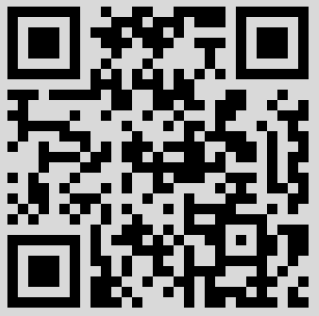


3. Пресмая Э.Л., Сояия И. М. Точки равновесия в обобщенной игровой задаче наилучшего выбора. - Теория вероятн. и ее примен., 1975, т. ХХ, в. 4, с. $770-$ 781.

4. Fushimi $M$. The secretary problem in a competitive situation. - J. Oper. Res. Soc. Japan, 1981, v. 24, p. 350-358.

5. Enns E. G., Ferenstein E. The horse game. - J. Oper. Res. Soc. Japan, 1985, v. 28, p. $51-62$.

6. Sakaguchi $M$. Some two person bilateral games in the generalized secretary problem. - Math. Japon., 1989, v. 34, p. 637-654.

Поступила в редакцию

31. VII.1996

(c) $1997 \mathrm{r}$.

МЕДНИЦКИЙ Ю. В.*

\title{
О СУЩЕСТВОВАНИИ СИЛЬНЫХ РЕШЕНИЙ ЛИНЕЙНЫХ СТОХАСТИЧЕСКИХ ДИФФЕРЕНЦИАЛЬНЫХ УРАВНЕНИЙ НА $\mathbf{R}^{\infty}$
}

\begin{abstract}
В работе доказывается существование сильного решения линейного стохастического дифференциального уравнения на $\mathbf{R}^{\infty}$. Используется бесконечномерная модификация метода последовательных приближений для решения систем частного вида, а также аналог метода Жордана приведения матрицы к клеточному виду. Показывается неединственность построенного решения.
\end{abstract}

Ключевые слова и фразы: стохастические уравнения.

Хорошо известно, что для всякого непрерывного линейного оператора $A$ в банаховом пространстве $X$ однозначно разрешимы обыкновенное дифференциальное уравнение

$$
x^{\prime}(t)=A x(t), \quad x(0)=x_{0},
$$

и стохастическое дифференциальное уравнение

$$
d x(t)=A x(t) d t+d w(t), \quad x(0)=x_{0},
$$

где $w(t)$ - произвольный винеровский процесс в $X$ (см. определение ниже).

Однако ситуация кардинально меняется для небанаховых локально выпуклых пространств (даже для пространств Фреше). Может случиться, что уравнения (1) и (2) не имеют никаких решений (кроме нулевого решения уравнения (1)), а также, что они разрешимы неоднозначно (см. примеры в [1]).

П р и м е p 1. Уравнение (1) в пространстве $\mathbf{R}^{\infty}$, соответствующее системе $x_{n}^{\prime}(t)=x_{n+1}(t), n \in \mathrm{N}$, имеет бесконечно много решений при любых началыных условиях. Действительно, пусть $x_{0}=\left(\alpha_{1}, \alpha_{2}, \ldots\right)$, тогда для любой бесконечно дифференцируемой функции $\varphi$ на прямой такой, что $\varphi^{(n-1)}(0)=\alpha_{n}$, отображение $t \mapsto\left(x_{n}(t)\right)=\left(\varphi^{(n-1)}(t)\right)$ будет являться решением этой системы. Ясно, что это решение всегда неединственно.

Пусть $H$ - сепарабельное гильбертово пространство, непрерывно и плотно вложенное в локально выпуклое пространство $X$. Тогда для всякого $k \in X^{*}$, где $X^{*}-$

*Московский государственный университет, механико-математический факультет, Воробъевы горы, 119899 Москва, Россия. 
топологически сопряженное $\mathrm{x} X$, ограничение $k$ на $H$ задается через представление Рисса в виде $\langle k, h\rangle=(j(k), h)_{H}$, где $j(k) \in H$.

O п р е д е л е и и е. Случайный процесс $w(t), t \geqslant 0$, со значениями в $X$ (определенный на нехотором вероятностном пространстве $\Omega$ ) называют винеровским процессом, ассоциированным с $H$, если для всякого $k \in X^{*} \mathrm{c}\|j(k)\|_{H}=1$ процесс $\langle k, w(t))$ является винеровским в обычном смысле (см. [2]).

Напомним, что процесс $x(t)$, являющийся решением уравнения (2), называют его сильным решением, если для каждого фиксированного $t \geqslant 0$ случайный элемент $x(t)$ измерим относительно $\sigma$-алгебры $\mathcal{F}_{t}$, порожденной элементами $w(s), s \leqslant t$. В случае, когда $X$ - банахово пространство, решение уравнения (2) будет сильным.

Мы будем рассматривать линейное стохастическое дифференциальное уравнение на $\mathbf{R}^{\infty}$ вида

$$
d x(t)=[A x(t)+f(t)] d t+d w(t),
$$

иногда записывая его в координатной форме, подразумевая при этом соответствующее интегральное уравнение

$$
x(t)=x(0)+\int_{0}^{t}(A x(s)+f(s)) d s+d w(t)
$$

где $A$ - элемент пространства $L\left(\mathbf{R}^{\infty}\right)$ линейных непрерывных операторов из $\mathbf{R}^{\infty}$ в $\mathbf{R}^{\infty}, f(t)$ - непрерывное отображение из $\mathbf{R}$ в $\mathbf{R}^{\infty}$.

В интересующем нас случае $X=\mathbf{R}^{\infty}$ полагаем $H=l^{2}$. Тогда для всякой последовательности независимых винеровских процессов $w_{n}(t)$ процесс $w(t)=\left(w_{n}(t)\right)_{n=1}^{\infty}$ является винеровским в смысле приведенного выше определения.

В [3] показано, что в пространстве $\mathbf{R}^{\infty}$ разрешимо всякое обыкновенное линейное неоднородное дифференциальное уравнение вида

$$
x^{\prime}(t)=A x(t)+f(t), \quad x(0)=x_{0},
$$

где $A$ и $f$ подразумеваются теми же, что и для уравнения (3).

Основной результат настоящей работы состоит в том, что уравнение (3) имеет сильное решение (однако, в отличие от банахова случая, такое решение не единственно). При доказательстве этого используется схема, аналогичная предложенной в [3], а при доказательстве предварительной леммы проводится бесконечномерная модификация метода последовательных приближений для решения системы частного вида.

Теорема. Уравнеяие (3) имеет сиљьное решение для произволькых оператора $A$ из $L\left(\mathbf{R}^{\infty}\right)$ и фучкции $f$ из $C\left(\mathbf{R}, \mathbf{R}^{\infty}\right)$.

3 а м е ч а н е 1. Предположение о справедливости данного утверждения было высказано в замечании 6.6 работы [1].

Лемма. Уравмеяие, имеющее в коордияаткой форме вид

$$
d x_{n}(t)=x_{n+1}(t) d t+d w_{n}(t)+f_{n}(t) d t, \quad x(0)=x_{0}
$$

имеет сильное решение для прочзвольной функиии $f$ из $C\left(\mathbf{R}, \mathbf{R}^{\infty}\right)$.

Д о к а з а т л ь ство. Для упрошения выкладок, производимых в ходе дальнейших рассуждений, процессы $x(t)$ и $w(t)$ предполагаются выходящими из нуля. Решение будем искать методом последовательных приближений, строя его по аналогии с конечномерным вариантом, описанным, например, в [2]. Для начала перепишем уравнение (4) в виде

$$
x_{n}(t)=\int_{0}^{t}\left(x_{n+1}(s)+f_{n}(s)\right) d s+w_{n}(t) .
$$


Последовательные приближения определим индуктивной формулой

$$
x_{n}^{(k+1)}(t)=\int_{0}^{t}\left(x_{n+1}^{(k)}(s)+f_{n}(s)\right) d s+w_{n}(t) .
$$

При этом в качестве нулевого приближения возьмем решение обыкновенного неоднородного дифференциального уравнения $\left[x_{n}^{(0)}(t)\right]^{\prime}=x_{n+1}^{(0)}(t)+f_{n}(t)$ с нулевым начальным условием. Существование этого решения доказано в [3].

Теперь оценим $\mathbf{E}\left|x_{n}^{(k+1)}(t)-x_{n}^{(k)}(t)\right|^{2}$. При $k=0$ имеем $\mathbf{E}\left|x_{n}^{(1)}(t)-x_{n}^{(0)}(t)\right|^{2}=$ $\mathbf{E}\left|w_{n}(t)\right|^{2}=t$. При $k>0$, применяя неравенство Коши-Буняковского, получаем:

$$
\begin{aligned}
& \mathbf{E}\left|x_{n}^{(k+1)}(t)-x_{n}^{(k)}(t)\right|^{2}=\mathbf{E}\left|\int_{0}^{t}\left(x_{n+1}^{(k)}(s)-x_{n+1}^{(k-1)}(s)\right) d s\right|^{2} \\
& \quad \leqslant t \cdot \mathbf{E}\left[\int_{0}^{t}\left(x_{n+1}^{(k)}(s)-x_{n+1}^{(k-1)}(s)\right)^{2} d s\right]=t \cdot \int_{0}^{t} \mathbf{E}\left|x_{n+1}^{(k)}(s)-x_{n+1}^{(k-1)}(s)\right|^{2} d s .
\end{aligned}
$$

Из этих оценок вытекает, что

$$
\mathbf{E}\left|x_{n}^{(k+1)}(t)-x_{n}^{(k)}(t)\right|^{2} \leqslant \frac{t^{2 k+1}}{2^{k} k !}
$$

и $x_{n}^{(k)}(t)$ сходится в среднем квадратичном к некоторому $x_{n}(t)$, причем равномерно на любом конечном отрезке, поскольку ряд из правых частей оценок (7) сходится. Докажем, что $x(t)=\left(x_{n}(t)\right)$ является решением уравнения (4) (эквивалентного (5)). Заметим, что процесс $x(t)$ прогрессивно измерим как предел прогрессивно измеримых случайных функций. Теперь перейдем к пределу в формуле (6). Левая часть будет сходиться к $x_{n}(t)$, а интеграл в правой части сходится в среднем квадратичном $\mathrm{K}$ соответствующему интегралу с $x_{n+1}(s)$ вместо $x_{n+1}^{(k)}(s)$ :

$$
\begin{aligned}
& \mathbf{E}\left|\int_{0}^{t}\left(x_{n+1}^{(k)}(s)+f_{n}(s)\right) d s-\int_{0}^{t}\left(x_{n+1}(s)+f_{n}(s)\right) d s\right|^{2} \\
& =\mathbf{E}\left|\int_{0}^{t}\left(x_{n+1}^{(k)}(s)-x_{n+1}(s)\right) d s\right|^{2} \leqslant t \cdot \int_{0}^{t} \mathbf{E}\left|x_{n+1}^{(k)}(s)-x_{n+1}(s)\right|^{2} d s .
\end{aligned}
$$

Последнее стремится к нулю в силу равномерной сходимости в среднем квадратичном $x_{n+1}^{(k)}(s)$ к $x_{n+1}(s)$ на отрезке $[0, t]$. Лемма доказана.

3 а м е ч а и и е 2. Уравнение (5) при каждом $\omega$ можно рассматривать как обыкновенное интегральное уравнение. В качестве такового оно будет иметь бесконечно много решений. Для того чтобы получить их, достаточно прибавить х построенному в лемме решению неоднородного уравнения любое решение однородного дифференциального уравнения из примера 1 . Получаемые таким образом решения могут оказаться, не согласованными с $\mathcal{F}_{t}$, т.е. не являющимися решениями стохастического уравнения. Таховыми, в частности, будут решения вида $x_{n}(\omega, t)=w_{1}(\omega, 1) \varphi^{(n-1)}(t)$, где $\varphi(t)$ берется из примера 1.

Дак а за те льс т в о т е о р м ы. Пусть $A \in L\left(\mathbf{R}^{\infty}\right)$. Тогда этот оператор задается матрицей $\left\{a_{i, j}\right\}(i, j \in \mathbf{N})$, причем $k$-я строка матрицы $a_{k}$ есть элемент $R_{\infty}$ - пространства последовательностей с конечным числом ненулевых членов. Пусть $e_{k} \in R_{\infty}$ - стандартные базисные векторы, а $\varphi_{k}$ - соответствующие координатные функционалы. Рассмотрим следующий итеративный процесс, аналогичный описанному в [3].

II а $\mathbf{r}$ 1. Положим $v_{1}^{1}=e_{1}$. Если $a_{1} \in \operatorname{Lin}\left(v_{1}^{1}\right)$, то полагаем $\xi_{1}^{1}(t)=w_{1}(t)$, $g_{1}^{1}=f_{1}, \lambda_{1, i}^{1}=0$ при $i \neq 1, \lambda_{1,1}^{1}$ таково, что $a_{1}=\lambda_{1,1}^{1} v_{1}^{1}$, и считаем, что шаг 
закоччен. Если же $a_{1} \notin \operatorname{Lin}\left(v_{1}^{1}\right)$, то полагаем $\xi_{1}^{1}(t)=w_{1}(t), g_{1}^{1}=f_{1}, \lambda_{1,2}^{1}=1, \lambda_{1, i}^{1}=0$ при $i \neq 2$ и $v_{2}^{1}=\sum_{k=1}^{\infty} \varphi_{k}\left(v_{1}^{1}\right) a_{k}=a_{1}$.

В этом случае шаг продолжается. Опишем индуктивно его продолжение. Пусть шаг не закончен и векторы $v_{i}^{1}$, числа $\lambda_{j, k}^{1}$, процессы $\xi_{j}^{1}(t)$, функция $g_{j}^{1}$ при $i \leqslant n$, $j \leqslant n-1, k \in \mathbf{N}$ построены. Если

$$
\sum_{k=1}^{\infty} \varphi_{k}\left(v_{n}^{1}\right) a_{k} \in M=\operatorname{Lin}\left(v_{1}^{1}, \ldots, v_{n}^{1}\right)
$$

то полагаем $\xi_{n}^{1}(t)=\sum_{k=1}^{\infty} \varphi_{k}\left(v_{n}^{1}\right) w_{k}(t), g_{n}^{1}(t)=\sum_{k=1}^{\infty} \varphi_{k}\left(v_{n}^{1}\right) f_{k}(t), \lambda_{n, i}^{1}=0$ при $i \geqslant n+1$, а при $i \leqslant n$ числа $\lambda_{n, i}^{1}$ таковы, что

$$
\sum_{k=1}^{\infty} \varphi_{k}\left(v_{n}^{1}\right) a_{k}=\sum_{i=1}^{n} \lambda_{n, i}^{1} \cdot v_{i}^{1}
$$

В этом случае считаем, что шаг окончен.

Если $\sum_{k=1}^{\infty} \varphi_{k}\left(v_{n}^{1}\right) a_{k} \notin M$, то полагаем $\lambda_{n, n+1}^{1}=1, \lambda_{n, i}^{1}=0$ при $i \neq n+1$, $\xi_{n}^{1}(t)=\sum_{k=1}^{\infty} \varphi_{k}\left(v_{n}^{1}\right) w_{k}(t), g_{n}^{1}(t)=\sum_{k=1}^{\infty} \varphi_{k}\left(v_{n}^{1}\right) f_{k}(t)$ и $v_{n+1}^{1}=\sum_{k=1}^{\infty} \varphi_{k}\left(v_{n}^{1}\right) a_{k}$. В этом случае шаг продолжается.

Пусть $N_{1}=\left\{n \in \mathrm{N}: v_{n}^{1}\right.$ определено $\}$. Рассмотрим уравнения:

$$
d y_{n}^{1}(t)=\sum_{j=1}^{\infty} \lambda_{n, j}^{1} y_{j}^{1}(t) d t+d \xi_{n}^{1}(t)+g_{n}^{1}(t) d t \quad\left(n \in N_{1}\right)
$$

Заметим, что эта система либо конечна, либо удовлетворяет условиям леммы и, следовательно, имеет решение $\left\{y_{n}^{1}(t): n \in N_{1}\right\}$. Она получена из исходной заменой переменных $y_{n}^{1}=\sum_{k=1}^{\infty} \varphi_{k}\left(v_{n}^{1}\right) x_{k}$.

Положим $L_{1}=\operatorname{Lin}\left\{v_{n}^{1}: n \in N_{1}\right\}, D_{1}=\left\{n \in \mathrm{N}: e_{n} \in L_{1}\right\}$. Отметим, что $v_{n}^{1}$ линейно независимы и что $e_{1} \in L_{1}, 1 \in D_{1}$. Если $D_{1}=N$, то процесс заканчивается на первом urare. В противном случае действуем дальше по той же схеме.

Опишем шаг процесса с номером $n$. Считаем, что перед его началом определены $N_{j}=\left\{i \in \mathbf{N}: v_{i}^{j}\right.$ определено $\}, 1 \leqslant j \leqslant n-1, L_{n-1}=\operatorname{Lin}\left\{v_{i}^{j}: i \in N_{j}, 1 \leqslant j \leqslant n-1\right\}$, $\left\{v_{i}^{j}: i \in N_{j}, 1 \leqslant j \leqslant n-1\right\}-$ линейно независимое подмножество $R_{\infty}, D_{n-1}=\{i \in$ N: $\left.e_{i} \in L_{n-1}\right\}$, причем $\left\{e_{1}, e_{2}, \ldots, e_{n-1}\right\} \subset L_{n-1}$.

III а г $n$. Положим $v_{1}^{n}=e_{m}$, где $m=\min \left\{k: k \notin D_{n-1}\right\}$. Пусть $k \in \mathbf{N}$ и $v_{i}^{n}$, $\lambda_{j, p}^{n}, \xi_{j}^{n}(t), g_{j}^{n}(t)$ при $i \leqslant k, j \leqslant k-1$ построены. Если

$$
\sum_{j=1}^{\infty} \varphi_{j}\left(v_{k}^{n}\right) a_{j} \in M=\operatorname{Lin}\left(L_{n-1} \cup\left\{v_{1}^{n}, \ldots, v_{k}^{n}\right\}\right)
$$

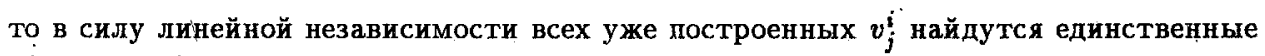
$\mu^{1}, \ldots, \mu^{n-1} \in R_{\infty}, \lambda_{k, 1}^{n}, \ldots, \lambda_{k, k}^{n} \in \mathbf{R}$ такие, что

$$
\sum_{j=1}^{\infty} \varphi_{j}\left(v_{k}^{n}\right) a_{j}=\sum_{i=1}^{k} \lambda_{k, i}^{n} v_{i}^{n}+\sum_{i=1}^{n-1} \sum_{p \in N_{i}} \varphi_{p}\left(\mu^{i}\right) v_{p}^{i}
$$


Полагаем $\lambda_{k, i}^{n}=0$ при $i>k, \xi_{k}^{n}(t)=\sum_{j=1}^{\infty} \varphi_{j}\left(v_{k}^{n}\right) w_{j}(t)$,

$$
\begin{aligned}
g_{k}^{n}(t)= & \sum_{j=1}^{\infty} \varphi_{j}\left(v_{k}^{n}\right) f_{j}(t)+\sum_{i=1}^{n-1} \sum_{p \in N_{i}} \varphi_{p}\left(\mu^{i}\right) y_{p}^{i}(t) \\
& +\sum_{\substack{1 \leqslant i<n \\
\left|N_{i}\right|=p<\infty}} \varphi_{p}\left(\mu^{i}\right)\left(g_{p}^{i}-\sum_{j=1}^{\infty} \varphi_{j}\left(v_{p}^{n}\right) f_{j}\right)
\end{aligned}
$$

и считаем, что шаг окончен.

Если $\sum_{j=1}^{\infty} \varphi_{j}\left(v_{k}^{n}\right) a_{j} \notin M$, то полагаем $\lambda_{k, k+1}^{n}=1, \lambda_{k, i}^{n}=0$ при $i \neq k+1$ и

$$
\begin{aligned}
v_{k+1}^{n} & =\sum_{j=1}^{\infty} \varphi_{j}\left(v_{k}^{n}\right) a_{j}, \quad \xi_{k}^{n}(t)=\sum_{j=1}^{\infty} \varphi_{j}\left(v_{k}^{n}\right) w_{j}(t) \\
g_{k}^{n}(t) & =\sum_{j=1}^{\infty} \varphi_{j}\left(v_{k}^{n}\right) f_{j}(t) .
\end{aligned}
$$

В этом случае шаг продолжается.

В итоге $n$-го шага мы получаем $N_{n}=\left\{k \in \mathrm{N}: v_{k}^{n}\right.$ определено $\}$. Рассмотрим систему уравнений:

$$
d y_{k}^{n}(t)=\sum_{j=1}^{\infty} \lambda_{k, j}^{n} y_{j}^{n}(t) d t+d \xi_{k}^{n}(t)+g_{k}^{n}(t) d t \quad\left(k \in N_{n}\right) .
$$

Эта система получается из исходной заменой переменных вида

$$
y_{k}^{n}=\sum_{i=1}^{\infty} \varphi_{i}\left(v_{k}^{n}\right) x_{i}
$$

Она либо конечна (в этом случае $g_{k}^{n}$ в последњем уравнении является некоторым случайным процессом), либо удовлетворяет условиям леммы и, следовательно, имеет peшrение $\left\{y_{k}^{n}(t): k \in N_{n}\right\}$. На этом шаг $n$ оканчивается.

Весь процесс состоит из конечного или счетного числа шагов. В итоге мы получаем, что определены $P=\left\{(n, k): v_{k}^{n}\right.$ определено $\} \subset \mathrm{N}^{2}, H=\left\{v_{k}^{n}:(n, k) \in P\right\}$ и $\left\{y_{k}^{n}:(n, k) \in P\right\}$. Очевидно, что $H-$ алгебраический базис в $R_{\infty}$, так ках множество $H$ ликейно независимо и $\operatorname{Lin}(H)$ содержит все $e_{m}$. Тогда для любого $m \in \mathbf{N}$ найдется единственный элемент $\mu_{m} \in R_{P}$ такой, что $e_{m}=\sum_{(n, k) \in P} \mu_{m, k}^{n} v_{k}^{n}$. Положим

$$
x_{m}(t)=\sum_{(n, k) \in P} \mu_{m, k}^{n} y_{k}^{n}(t)
$$

Из построения $y_{k}^{n}$ вытекает, что процесс $x(t)=\left(x_{m}(t)\right)$ прогрессивно измерим и удовлетворяет уравнению (3). Теорема доказана.

3 а м е ч а н и е 3. Дохазанная теорема существования решения уравнения (3) не затрагивает вопроса о его единственности. Поскольку разность цвух решений уравнеиия (3) является при каждом $\omega$ решением однородного уравнения (1) с нулевым начальным условием, то единственность решения уравнения (3) эквивалентна единственности решения уравнения (1) с $x_{0}=0$.

Пусть $x(\omega, t)$ - решение уравнения (3), $z(t)$ - решение уравнения (1) с $x_{0}=0$. Тогда для произвольной измеримой функции $\xi(\omega)$ решением (3) будет язляться также и $x(\omega, t)+\xi(\omega) z(t)$. Таким образом могут быть получены и несогласованные решения, 
например, при $\xi(\omega)=w_{1}(\omega, 1)$. Из этого вытекает, что однозначная разрешимость уравнения (3) равносильна однозначной разрешимости уравнения (1) и имеет место тогда и только тогда, когда все решения (3) являются согласованными. Отметим, что однозначная разрешимость (1) для $A \in L\left(\mathbf{R}^{\infty}\right)$ равносильна (см. [1, пр. 4.24]) существованию $\exp (t A)$ для всех $t$. Согласно [4], последнее имеет место, если спектр $A$ в произведении комплексных прямых не более чем счетен.

3 а м е ч а н и е 4. Доказанная теорема показывает разрешимость уравнения (3) для «естественного» винеровского процесса в $\mathbf{R}^{\infty}$. Однако остается открытым вопрос, верен ли этот результат для произвольного винеровского процесса в $\mathbf{R}^{\infty}$ (в смысле приведенного выше определения). «Естественность» винеровского процесса существенно используется лишь при выводе оценок (7). Это позволяет надеяться на то, что утверждение теоремы остается справедливым и в общем случае.

3 а м е ч а н и е 5. Отметим, что в [1] приведены примеры неразрешимых линейных стохастических дифференциальных уравнений в пространствах Фреше. Неизвестно, вытекает ли из разрешимости всех уравнений (1) в пространстве $X$ разрешимость каждого уравнения (2) (хотя бы при некотором выборе $H$ и соответствующем выборе винеровского процесса). Аналогичный вопрос открыт и для индивидуальных операторов - влечет ли разрешимость уравнения (1) с фиксированным $A$ (для всех $x_{0}$ ) разрешимость уравнения (2) для какого-нибудь выбора $H$ и $w(t)$ (а может быть, и для любого их выбора). При некоторых дополнительных условиях (типа существования непрерывного разрешающего семейства $S_{t}: X \rightarrow X$ уравнения (1)) положительный ответ на последний вопрос дан в [1].

3 а м е ч а н и е 6. Имеется ряд достаточных условий разрешимости нелинейных уравнений в $\mathbf{R}^{\infty}$ вида

$$
d x(t)=d w(t)+a(t, x(t)) d t
$$

однако они не охватывают произвольных линейных уравнений (см. [1]). Например, в [5] требуется, чтобы $a$ принимало значения в $l^{2}$ (и при этом существенно зависело лишь от начальных компонент вектора $x(t)$ ). При этих предположениях доказывается существование слабото решения уравнения (8). Можно проверить, что всякий непрерывный линейный оператор $A: \mathbf{R}^{\infty} \rightarrow l^{2}$ конечномерен.

\section{СПИСОК ЛИТЕРАТУРЫ}

1. Bogachev V.I. Deterministic and stochastic differential equation in infinite-dimensional spaces. - Acta Appl. Math., 1995, v. 40, p. 25-93.

2. Веятиель А. Д. Курс теории случайных процессов. М.: Наука, 1975, 319 с.

3. Шкария $C$. $A$. Несколько результатов о разрешимости обыкновенных линейных дифференциальных уравнений в локально выпуклых пространствах. - Матем. с6., 1990, т. 181, № 9, с. 1183-1195.

4. Lemmert $R$., Weckbach $\ddot{A}$. Charakterisierungen zeilenendlicher Matrizen mit abzählbaren Spektrum. - Math. Z., 1984, v. 188, № 1, p. 119-129.

5. Jerschow $M$. Infinite-dimensional Wiener processes with drift. - Stochastic Process. Appl., 1994, v. 52, p. 229-238. 